\title{
Search for Astronomical Neutrino from Blazar TXS0506+056 in Super-Kamiokande
}

\author{
Kaito Hagiwara*, For the Super-Kamiokande Collaboration \\ Okayama University, Faculty of Science, Okayama 700-8530, Japan \\ E-mail: E.hagiwarads.okayama-u.ac.jp
}

\begin{abstract}
Astronomical neutrinos from the direction of the blazar TXS0506+056 were searched in the Super-Kamiokande (SK). A high-energy neutrino event that seems to originate from the blazar was observed in the IceCube experiment at September 2017. Since SK is the largest water Cherenkov detector in the $\mathrm{MeV}$ to $\mathrm{TeV}$ region, there is a possibility of observing the astronomical neutrino events from the blazar. The experimental data from SK-I to SK-IV spanning a period from April 1996 to February 2018 were used. All events were classified by the interaction point and the energy. The upward-going muon sample was explored within 5 degrees around the location of the blazar. The fully-contained and partially-contained samples within a 10 degrees search circle were used. No significant excess was found in all types of events from the KolmogorovSmirnov test.
\end{abstract}

36th International Cosmic Ray Conference -ICRC2019-

July 24th - August 1st, 2019

Madison, WI, U.S.A.

\footnotetext{
* Speaker.
} 


\section{Introduction}

The IceCube Neutrino Observatory detected a high-energy neutrino with an energy of about $290 \mathrm{TeV}$ on 22 September 2017 at 20:54:30.43 Coordinated Universal Time [四][]. The information of the event (IceCube-170922A) was shared via the Gamma-ray Coordinate Network (GCN) [B]. The neutrino arrival direction was soon observed by multiple observatories covering a broad energy range. The IceCube-170922A was coincident in location of blazar TXS0506+056 and coincident in time with a gamma-ray flare that started from April 2017. TXS0506+056, a blazar of $\mathrm{BL}$ Lac type, is located at right ascension $(\mathrm{RA})=77.3582^{\circ}$ and declination $(\mathrm{Dec})=+5.6931^{\circ}(\mathrm{J} 2000$ equinox) [ [G] and the redshift is $z=0.3365 \pm 0.0010$ [G]. Furthermore the IceCube group found another excess of high-energy neutrino event from that direction between September 2014 and March 2015[[]]. The state of enhanced gamma-ray activity, however, was not observed during that period. This suggests the blazars are one of the sources of the high-energy neutrino.

The Super-Kamiokande (SK) [6] is a large water Cherenkov detector which is located at 1000 $m$ underground (2700 m.w.e.) in the Kamioka-mine, Japan. Since SK is sensitive to atmospheric neutrinos in the energy range from about $30 \mathrm{MeV}$ to few tens $\mathrm{TeV}$, it is possible to search for a lower energy region than IceCube. Furthermore, due to its underground location, SK is not suffering from an high rate of cosmicmuon background. The average rate of cosmic ray muons is about $3 \mathrm{~Hz}$, which is a factor $10^{-5}$ smaller than at the surface. The neutrino direction can be determined with good accuracy depending on the type of reaction $\left(5^{\circ}\right.$ or $\left.10^{\circ}\right)$ in the energy region above few GeV.

Neutrinos coming from the direction of TXS0506 were searched in SK using all events from April 1996 to February 2018. Atmospheric neutrino events were estimated using Monte Carlo simulation as the background events. High angular resolution was achieved by setting a high energy threshold. The obtained results were evaluated as Fluence and Energy-Flux.

\section{Super-Kamiokande}

The SK detector constructed a cylindrical tank, $39.3 \mathrm{~m}$ diameter and $41.4 \mathrm{~m}$ tall, has a 50 kilotonne (22.5 kilotonne fiducial) pure water as neutrino target. The tank is separated into an inner detector (ID) volume with 11129 photomultiplier tubes (PMTs) and an outer detector (OD) volume with 1885 PMTs. Between the ID and the OD is the support structure with a thickness of about $55 \mathrm{~cm}$. To improve the light sensitivity of the OD PMTs, the outside wall of the support structure is surrounded by a Tyvek sheet. One the other hand, the inside wall of the support structure is surrounded by a black sheet of the polyethylene terephthalate to reduce the reflection of the light.

SK started operation in April 1996 and has observed neutrinos with four periods (SK-I, -II, -III, and -IV). The SK-I period data has been took from April 1996 to July 2001 (1489.2 live-time day) with $40 \%$ photocoverage of ID. The SK-II operated between December 2002 and October 2005 (798.6 live-time day) with a photocoverage of $19 \%$ because of an accident that half of the ID PMTs were destroyed in November 2001. After the all PMTs restored in July 2006, SK-III period started with 40\% photocoverage until September 2008 (518.1 live-time day). SK-IV period, with new detector electronic systems, continued until June 2018 when the refurbishment of SK tank 
began. In this paper, SK-IV data are used up to February 2018 with 3118.5 live-time days. For the analysis, the high-energy neutrino data of SK-I to SK-IV are used. Total live-time is 5934.4 days.

\section{Analysis}

There are three classifications for the atmospheric neutrino events observed in SK. The neutrinos interacted inside the fiducial volume, whose daughter particles are then detected as Cherenkov rings by the ID PMTs. Such neutrino events can be classified into two categories. The event that all daughter particles stop within the ID is categorized as fully-contained (FC), whereas it is categorized as partially-contained (PC) if any of the produced particles exit to the OD and leave signals there. The energetic muon-neutrinos interacting with the rock around the SK create muons which enter the ID through OD. Only the muons with upward momentum are categorized as upwardgoing muon (UPMU) because down-going muon events of this kind could not be distinguished from cosmic muons.

The reduction cuts were done to identify three types of events. For FC and PC events, the reconstructed vertex was required to be more than $2.0 \mathrm{~m}$ away from the ID surface, which is defined as fiducial volume (22.5 kilotonnes). The visible energy in the detector was required to be greater than $30 \mathrm{MeV}$ for FC events and greater than $350 \mathrm{MeV}$ for PC events. If the number of hit PMTs in the OD was fewer than 16 hits (10 hits for SK-I) it is categorized FC events, otherwise it is PC events. The UPMU events selection cuts ensured that the reconstructed direction was below the horizon. As an event that passing through the detector, the fitted track length of $7.0 \mathrm{~m}$ or more was required. As an event that stopping in the detector, the fitted momentum of the muon of $1.6 \mathrm{GeV}$ or more was required. The Monte Carlo (MC) simulation of atmospheric neutrino events was also performed. It was generated and analyzed 500 years of live-time for each SK phase.

Since the scattering angle of lepton particle becomes larger for lower energies, a high energy threshold was set to achieve high angular resolution. From MC dataset, the angle between the incoming neutrino direction and reconstructed direction was calculated. By setting the threshold to 5.1 GeV for FC events, $1.8 \mathrm{GeV}$ for PC, and $1.6 \mathrm{GeV}$ for UPMU events, $68 \%$ or more of the data points were achieved within 10 degrees for FC and PC events, and within 5 degrees for UPMU events. An explicit upper energy cut was not applied.

\section{Result}

Figure $\square$ shows the sky-map of the selected neutrino events. The number of neutrino event rate depends on declination. Especially, the UPMU data sample have no events at declination above $54^{\circ}$. This is due to the fact that only neutrinos coming from below the horizon can be classified as UPMU. The number of observed in this search cone ( $\left.N_{\text {data }}\right)$ was 18, 29, 20 for FC, PC, and UPMU events, respectively, during SK I-IV.

Since the atmospheric neutrinos are background events in this analysis, it is necessary to estimate the observables of atmospheric neutrino in SK using MC. This MC code was made by Geant3[四] simulation toolkit to simulate the secondary particle interactions and tracking. The information of neutrino interaction was used the models named NEUT[[]]. The simulated atmospheric neutrino events for 500 years were created for each SK phase. The MC sample for each phase 

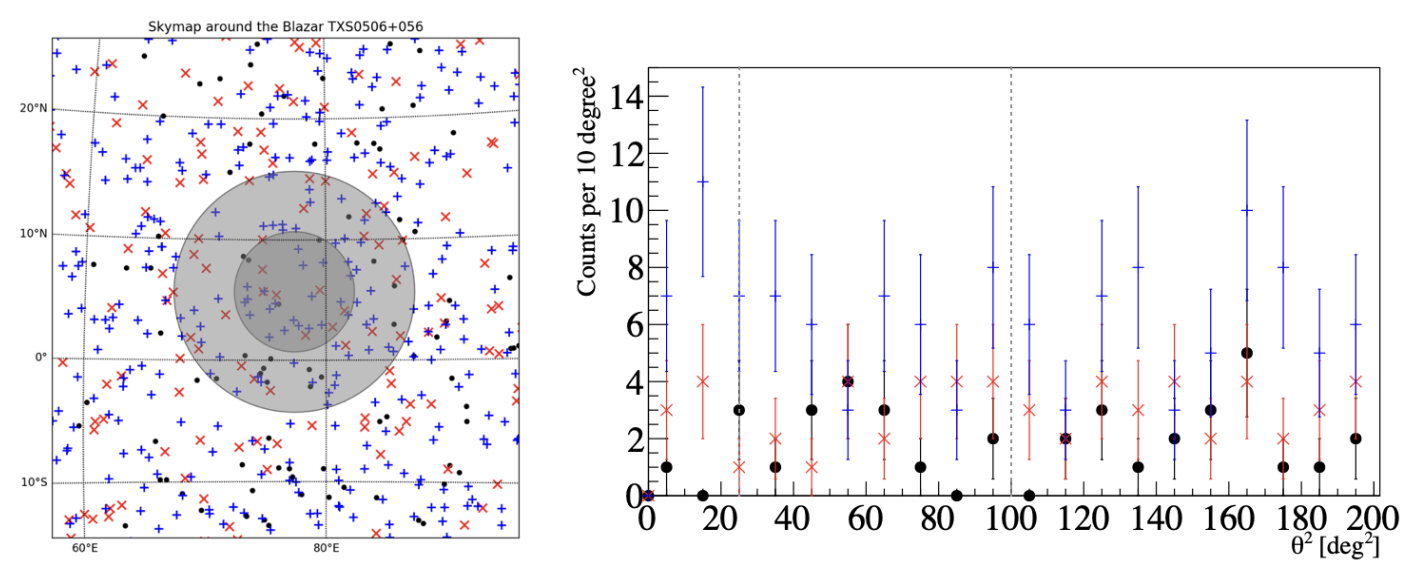

Figure 1: The selected FC (black circle), PC (red x), and UPMU (blue + ) events are shown. Left figure shows the location of blazar TXS0506+056 $(\alpha, \beta)=\left(77.3582^{\circ},+5.6931^{\circ}\right)$ in equatorial coordinates with right ascension on the $\mathrm{x}$-axis and declination on the $\mathrm{y}$-axis. The dark grey circle has a radius of 5 degrees and is a search area for UPMU events. The radius of the light grey circle is 10 degrees, which is the search area for FC and PC events. The right figure is preliminary result that the angle between location of blazar and experimental data points in the form of a square of degrees. The dashed line shows 5 degrees and 10 degrees.

were normalized by appropriate live-time, and then summed them together. Since the MC sample does not have an information of time, MC events were assigned right ascensions assuming a flat local sidereal time. The combined sample was then scaled on an event-by-event basis to the all-sky, which accounts for the flux normalization and neutrino oscillations.

Figure $\square$ shows the result of expected background and number of observed events. The experimental data agrees with the expected background within $0.7 \sigma$ for FC, $1.1 \sigma$ for PC, and $1.2 \sigma$ for UPMU events. The FC and PC events extend to all declinations and have a slightly positive slope due to the neutrino oscillation in the upward-going neutrinos. The double-bump structure around dec. $=-50,50$ can be seen due to the atmospheric neutrinos coming from near the horizon. As cosmic rays are more likely to interact due to the fact that the atmosphere is thicker and the path length to traverse this area is longer, cosmic muons are more likely to decay. Since the UPMU events require that the neutrinos come from below the horizon, the events with a declination of 54 degrees or more are not observed.

The expected background events $\left(N_{\mathrm{bkg}}\right)$ at the declination of blazar were 15.20, 22.91, and 14.57 for FC, PC, and UPMU events respectively from MC data. The same analysis was performed at the same declination and changed right ascension by 20 degree for the FC and PC events, and by 10 degree for UPMU events. The relative difference $\left(N_{\text {data }}-N_{\mathrm{bkg}}\right) / N_{\mathrm{bkg}}$ of each point was calculated. As the result, the neutrino events coming from the direction of the blazar agrees even in comparison with other direction at the level of $0.5 \sigma, 1.6 \sigma$, and $1.5 \sigma$ in FC, PC, and UPMU events.

The Kolmogorov-Smirnov test (KS-test) was performed to confirm the presence or absence of significant time-dependent events. If there is a relationship between the gamma-ray flare of the blazar and emission of neutrino, the neutrino flux should increase depending on time. The 10,000 pseudo datasets assuming that the event rate is constant for each SK phase were created 

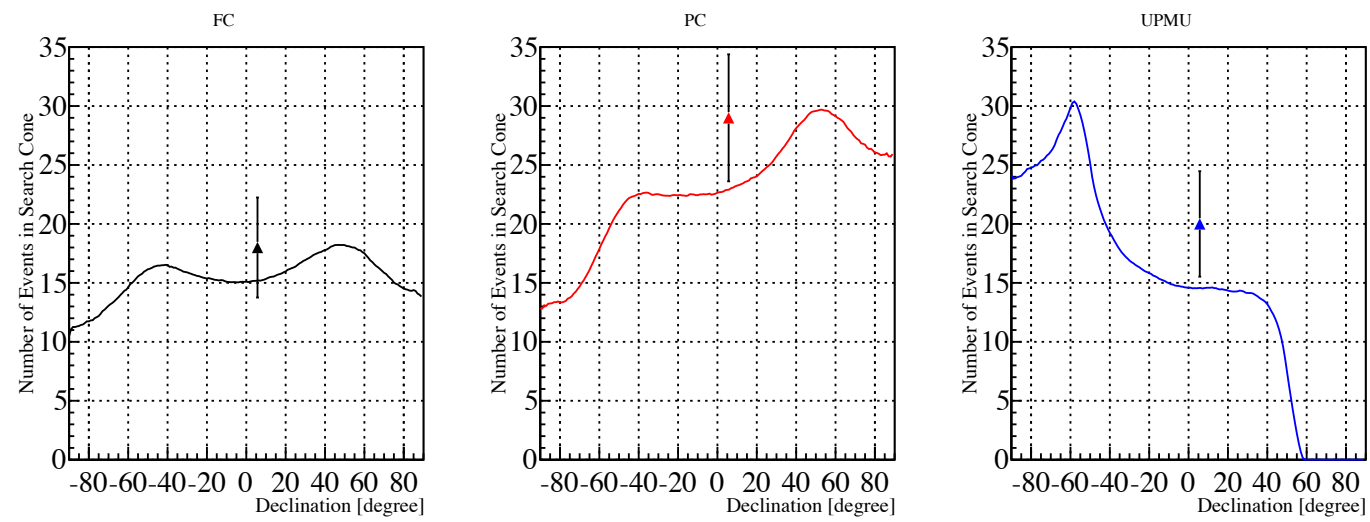

Figure 2: The number of expected events in the search area (solid line) for FC (black), PC (red), and UPMU (blue) are shown with the number of detected events in the search area from the data (point). The error shown here are $\sqrt{N_{\text {data }}}$

\begin{tabular}{cccc} 
& FC Event & PC Event & UPMU Event \\
\hline Observed Events & 18 & 29 & 20 \\
Expected Background & 15.20 & 22.91 & 14.57 \\
90\% C.L. Events & 10.19 & 14.62 & 12.69
\end{tabular}

Table 1: There is preliminary list of 90\% C.L. of neutrino events coming from the blazar TXS0506+056 direction.

by toy MC. The toy MC takes into consideration the efficiency depending on the neutrino arrival direction, the difference in the detection efficiency among SK phases, and the live-time. Since the accumulated events increase linearly if the dataset created in this way is uniformly distributed, the point having the largest distance from the linear increase is determined for each pseudo dataset. For the maximum distance distribution obtained from this, the ratio of pseudo data with the maximum distance larger than the maximum distance obtained from experimental data was calculated as the P-value of KS-test. The results were $61.08 \%, 41.17 \%$, and $98.33 \%$ for FC, PC, and UPMU events. There was no one that meets the significant level (P-value $<5 \%)$ to reject the assumptions. Therefore, it can be said that there is no significant time-dependent event.

From the results of event rate calculation and KS-test, no significant signal from blazar direction was found. Therefore, the number of $90 \%$ Confidence Level (C.L.), $N_{90}$, was determined from the number of observed events against the expected number of background events using a Poisson

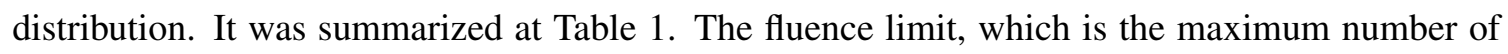
neutrinos per unit area observed at SK can be determined[可 [ए]]. Here, the only method of the calculation of fluence limit is described. The energy region was determined according to the method described in Ref.[W], which for FC and PC is in the range of $10 \mathrm{GeV}$ from the threshold and for UPMU is $1.6 \mathrm{GeV}$ to $10 \mathrm{TeV}$. 
For the FC and PC events, the neutrino fluence can be calculated using flowing equation.

$$
\Phi_{\mathrm{FC}, \mathrm{PC}}=\frac{N_{90}}{N_{T} \int \mathrm{d} E_{v} \sigma\left(E_{v}\right) \varepsilon\left(E_{v}\right) \lambda\left(E_{v}^{-2}\right)},
$$

where $N_{T}$ is the number of nucleons in the 22.5 kilotonnes of water used as neutrino target, $\sigma$ is the total cross-section for all interactions, $\varepsilon$ is the efficiency for measuring the neutrino event in the fiducial volume, and $\lambda$ is the number density of the neutrino events for a given energy spectrum with index of -2 . The fluence limits were calculated separately for each neutrino flavors by considering the cross-section and detection efficiency.

For the UPMU events, the neutrino fluence is calculated as flowing.

$$
\Phi_{\mathrm{UPMU}}=\frac{N_{90}}{A_{\mathrm{eff}}(z) \int \mathrm{d} E_{v} P\left(E_{v}\right) S\left(z, E_{v}\right) \lambda\left(E_{v}^{-2}\right)}
$$

where $A_{\text {eff }}$ is the effective area of SK, $P$ is the probability for a neutrino to create a muon, $S$ is the shadowing of the neutrinos due to interactions in the Earth, and $\lambda$ is the number density of the neutrino events for a given energy spectrum with index of -2 . $z$ represents the zenith angle of incoming neutrino, and $A_{\text {eff }}$ and $S$ depend on the zenith angle.

\section{Conclusion}

We performed a search for Super-Kamiokande neutrino detections coincident in the direction of blazar TXS0506+056, which is located at RA 77.3582 and Dec $+5.6931^{\circ}$. We used SK data taken from April 1996 to February 2018 to determine if there was any excess of events in search cone using Poisson statistics. In the few $\mathrm{GeV}$ to few tens $\mathrm{TeV}$ region of energy, high energy neutrino samples can be classified into fully-contained (FC), partially-contained (PC), and upward-going muon (UPMU) were searched. The search area around the blazar was 5 degrees for UPMU and 10 degrees for FC and PC events.

The background events were estimated by using the MC simulation of atmospheric neutrino for 500 years in each SK phase. By comparing the expected background with the experimental data, no significance excess was observed and the event rate agrees within 1.6 $\sigma$. Furthermore from the results of the Kolmogorov-Smirnov test, no significant temporal event excess was found from blazer direction. The 90\% C.L. events number were 10.19, 14.62, and 12.69 for FC, PC, and UPMU.

\section{References}

[1] The IceCube Collaboration, Science 361, Issue 6398 (2018), eaat1378.

[2] The IceCube Collaboration, Science 361, 147-151 (2018).

[3] GCN/AMON Notice, https://gcn.gsfc.nasa.gov/amon.html.

[4] E. Massaro et al., Astrophys. Space Sci. 357, 75 (2015).

[5] S. Paiano, R. Falomo, A. Treves, R. Scarpa, Astrophys. J. 854, L32 (2018).

[6] S. Fukuda, et al., Nucl. Instrum. Meth. A501:418-462 (2003). 
[7] R. Burn, et al., CERN-DD-EE-84-1 (1987).

[8] Y. Hayato, AcPPB 402477 (2009).

[9] M.E.C Swanson, et al., ApJ. 652, 206 (2006).

[10] E. Thrane, et. al., ApJ. 704, 503 (2009).

[11] Y. Ashie, et al., Phys. Rev. D71, 112005 (2005). 